\title{
High Intermediate Risk Pulmonary Embolism: The Use of Very Low Dose Catheter-Directed Ultrasound- Accelerated Thrombolysis
}

\author{
Lee W Greenspon ${ }^{1 *}$, Ryan D Reber ${ }^{1}$, Ravi Y Desai ${ }^{1}$, Theodore J Plush ${ }^{1}$, Matthew Hegewald ${ }^{2}$, \\ Haresh D Rochani ${ }^{4}$, Peter A Villas ${ }^{3}$ and Joseph Bonn ${ }^{3}$ \\ ${ }^{1}$ Division of Pulmonary \& Critical Care Medicine, Lankenau Medical Center, USA \\ ${ }^{2}$ Division of Radiology, Bryn Mawr Hospital, USA \\ ${ }^{3}$ Division of Radiology, Main Line Health, USA \\ ${ }^{4}$ Division of Biostatistics, Jiann-Ping Hsu College of Public Health, Georgia Southern University, USA \\ *Corresponding author: Lee Greenspon, Suite 230, MOB West, 100 Lancaster Avenue, Wynnewood, USA
}

\section{ARTICLE INFO}

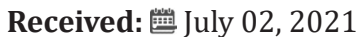

Published: 幽 August 12, 2021

Citation: Lee W Greenspon, Ryan D Reber, Ravi Y Desai, Theodore J Plush, Matthew Hegewald, et al., High Intermediate Risk Pulmonary Embolism: The Use of Very Low Dose Catheter-Directed UltrasoundAccelerated Thrombolysis. Biomed J Sci \& Tech Res 38(1)-2021. BJSTR. MS.ID.006094

Abbreviations: PE: Pulmonary Embolism; TPA: Tissue Plasminogen Activator; USAT: Ultrasound Assisted Thrombolysis; PAP: Pulmonary Artery Pressure; RVD: Right Venticular Dysfunction; CTEPH: Chronic Thromboembolic Pulmonary Hypertension; DVT: Deep Vein Thrombosis; ICH: Intracranial Hemorrhage; IVC: Inferior Venacava; PTT: Partial Thromboplastin Time

\section{ABSTRACT}

Objective: To determine the hemodynamic benefit, clot burden reduction and safety utilizing an ultra-low dose of Tissue Plasminogen Activator (TPA) with Catheter directed Ultrasound accelerated thrombolysis (USAT) in the management of high Intermediate Risk Pulmonary Embolism.

Design: Retrospective, observational case series.

Setting: Single Center Academic Community Hospital.

Patients: Fifty-two consecutive patients (27 males) with high Intermediate Risk PE treated with USAT were analyzed.

Measurements and Main Results: Forty-nine patients underwent bilateral USAT with $0.5 \mathrm{mg} / \mathrm{hr} /$ catheter of TPA (unilateral: $1.0 \mathrm{mg} / \mathrm{hr}$ ). Mean duration of therapy was $22.08+4.90$ hours. Changes in pulmonary artery pressure (PAP), Clot obstruction index by Miller score and procedure related bleeding complications were assessed. Paired t-testing was used to determine significance Mean RV/LV ratio was $1.52+0.36$. Miller score decreased $55.0 \%$ (mean $19.7+3.8$ to $8.9+4.7$; p value $<0.0001$ ). The systolic PAP decreased $7.3 \%$ from $63.6+15.1 \mathrm{mmHg}$ to $59.0+17.5 \mathrm{mmHg}$ ( $\mathrm{p}$ value $=0.0045$ ). The mean PAP decreased 5.4\% from $37.5+8.0 \mathrm{mmHg}$ to $35.5+9.1 \mathrm{mmHg}$ ( $\mathrm{p}$ value $=0.0097$ ). No procedural or bleeding related complications occurred.

Conclusion: Low dose USAT with $0.5 \mathrm{mg} / \mathrm{hr} /$ catheter with TPA for 24 hours is highly effective in reducing clot burden and PAP without bleeding or procedural related complications in high Intermediate Risk PE.

Keywords: Pulmonary Embolism; Thrombolysis; Ultrasound Accelerated Thrombolysis

\section{Introduction}

Outcomes in acute pulmonary embolism (PE) vary substantially depending on the presences or absence of hypotension [1]. In massive, High-Risk PE complicated by cardiogenic shock or the need for cardiopulmonary resuscitation, in-hospital mortality rates are $25 \%$ and $65 \%$, respectively [2,3]. Intermediate Risk PE represents a group characterized by the presence of right ventricular dysfunction 
(RVD) without systemic hypotension [2,4]. Estimates of in-hospital mortality for such patients have been as low as $2-3 \%$ as reported in a recent multicenter trial [5]. Despite the overall low mortality rate, $5-25 \%$ will demonstrate hemodynamic and clinical worsening requiring hemodynamic support and/or rescue thrombolysis $[5,6]$. Substantial variability exists in clinical outcomes in Intermediate Risk PE. In patients with RVD on echocardiogram combined with elevated troponin I mortality rates have been reported between 8-15\% [7,8]. Patients with RVD and concomitant lower extremity deep venous thrombosis (DVT) have a PE-related mortality of nearly 20\%, approximating that of massive, High-Risk PE [7]. In addition to mortality, persistent RVD and the development of chronic thromboembolic pulmonary hypertension (CTEPH) is substantially higher following a submassive Intermediate risk PE [9-12].

Patients who have an initial pulmonary artery systolic pressure over $50 \mathrm{mmHg}$ are at significant risk $[13,14]$. While systemic thrombolysis has been shown to reduce mortality in High-Risk PE [15], use in Intermediate Risk PE has been limited due to major bleeding complications, including intracranial hemorrhage (ICH) [5]. Recently, catheter-directed ultrasound-accelerated thrombolysis (USAT) has been developed as a treatment option for Intermediate Risk PE [4,16-24]. USAT has been shown to rapidly reverse pulmonary hypertension (PH) and RVD by significantly reducing pulmonary clot burden without major bleeding complications [4,16-24]. Additionally, there is increasing evidence that early clot reduction improves hemodynamic parameters and reduces persistent RVD $[9,18,19]$. Despite increasing clinical experience with USAT, variability exists in thrombolytic dosing and duration of therapy [4,16-24]. The purpose of this retrospective, single-center study, is to further describe the use of USAT, utilizing a lower dose thrombolytic protocol than previously reported and its impact on pulmonary artery pressure, clot burden and safety in high Intermediate Risk PE. In addition, we define high Intermediate Risk PE as those at greatest risk for hemodynamic deterioration, death, or the development of CTEPH.

\section{Material and Methods}

\section{Study Design and Patients}

A retrospective study of patients admitted with high Intermediate-Risk PE treated with USAT between July 2012 and February 2016 was performed. The study was conducted at a single academic center with approval from the Lankenau Medical institutional review board. (IRB\# F/N-R14-3414L). During the study period, 52 patients (27 males) were treated with USAT utilizing the EkoSonic Endovascular System (EKOS®) (EKOS Corporation, Bothell, Washington, United States of America) with recombinant tissue plasminogen activator (r-tPA) and concurrent intravenous (IV) unfractionated heparin infusion. Informed consent was obtained in all patients prior to undergoing USAT. PE was diagnosed in all patients by computerized tomography (CT) angiography with symptom onset $<2$ weeks. Intermediate Risk PE was defined as the presence of RVD without systemic hypotension $[2,4]$. RVD was detected utilizing electrocardiogram, cardiac biomarkers, CT scan, and transthoracic echocardiography.

High Intermediate Risk PE was considered those at greatest risk for acute hemodynamic deterioration, death or subsequent development of CTEPH (Table 1). All Patients had Right ventricular dilation on CT (Right Ventricular/Left Ventricular (RV/LV) maximum diameter ratio $>0.9$ on standard axial views. In addition, by our protocol, patients had elevation of troponin I (>0.05 ng/ $\mathrm{mL})$ and/or B-type natiuretic peptide $(>90 \mathrm{pg} / \mathrm{ml})$ and a proximal DVT or estimated right ventricular systolic pressure (RVSP) on echocardiogram $>50 \mathrm{mmHg}$. Lower extremity Doppler ultrasound was performed in the majority of patients to exclude deep venous thrombosis (DVT). If lower extremity DVT was present, retrievable inferior vena cava (IVC) filter was placed at the discretion of the treating physician. Patients were deemed candidates for USAT if they met clinical criteria for acute high Intermediate risk PE and had evidence of thrombus within the main or lobar pulmonary arteries on CT. The Department of Interventional Radiology performed USAT. All patients were admitted to the medical intensive care unit upon diagnosis and during USAT treatment.

\section{Standardized Procedure of Catheter-Directed Ultrasound-Accelerated Thrombolysis}

All patients were treated with the use of EkoSonic MACH4e Endovascular System (EKOS $囚$ ). The EKOS® system consists of a removable microsonic device that transmits microsonic energy into the clot. The insertion of the EkoSonic Endovascular System was performed under aseptic technique with continuous cardiac monitoring. Following central venous access, IVC venography was performed to ensure no thrombosis was present. IVC venography was incorporated in our protocol in 2015. Subsequently, left, right and main pulmonary arterial pressures were obtained. Selective left and right pulmonary arteriography was performed to determine clot burden and pulmonary arterial perfusion. The main and lower lobe pulmonary arteries were considered for catheter insertion only. Bilateral device placement was performed when emboli were located in both main or proximal lower lobe arteries. A continuous infusion of r-tPA was then initiated at $0.5 \mathrm{mg} / \mathrm{hr}$ through each catheter for bilateral treatments $(1.0 \mathrm{mg} / \mathrm{hr}$ for unilateral catheters). After successful device placement, patients were transferred to the medical intensive care unit for continuous monitoring. Treatment was continued for up to 24 hours, at which time the EKOS® devices were exchanged for angiographic catheters, and repeat pulmonary arteriography was performed in the interventional radiology suite to reassess clot burden, pulmonary arterial perfusion, and pulmonary arterial pressures. The introducer sheath(s) were removed, and hemostasis was obtained by manual compression. 


\section{Anticoagulation Therapy}

IV unfractionated heparin was utilized in all patients prior to, during, and immediately following USAT. Prior to undergoing USAT, unfractionated heparin was administered utilizing a standard weight-based nomogram. Activated partial thromboplastin time (aPTT) was monitored with a therapeutic target range of 68-101 seconds. During USAT, a low dose unfractionated heparin protocol was instituted. The rate of heparin infusion was adjusted to achieve and maintain an aPTT between 40-60 seconds. Following catheter removal, the standard weight-based nomogram was resumed, targeting an aPTT of 68-101 seconds. Subsequent anticoagulation was left to the discretion of the treating physician.

\section{Primary Outcomes}

The primary outcomes assessed in this study were the change in pulmonary artery pressure and extent of angiographic reduction in clot burden utilizing the Miller score [25] immediately following USAT. Pulmonary arterial pressures were obtained at the time of catheter insertion and catheter removal. Miller scores were determined from the pulmonary angiograms performed at the time of catheter insertion and catheter removal. Two diagnostic radiologists interpreted and scored the pulmonary angiograms independently. The Miller scores were averaged for the analysis.

\section{Secondary Outcomes}

The secondary outcomes assessed included bleeding, procedural related complications and all cause in-hospital mortality. Major bleeding complications were defined as ICH or bleeding severe enough to warrant cessation of therapy or blood product transfusion. Minor bleeding complications were defined as bleeding manageable with local compression, sheath upsizing, or unfractionated heparin and/or thrombolytic dose reduction [8]. Bleeding complications were assessed during USAT and up to 72 hours following catheter removal.

\section{Statistical Analysis}

Paired t-test was performed to test the hypotheses that the pre-USAT mean pulmonary artery pressure, pre-USAT pulmonary artery systolic pressure and pre-USAT Miller score were equal to the post-USAT mean pulmonary artery pressure, post-USAT pulmonary artery systolic pressure and post-USAT Miller score, respectively. The normality assumption for performing paired t-test was satisfied for all three comparisons by using kurtosis, skewness and shapiro-wilk test.

\section{Results}

\section{Clinical Characteristics}

During the study period, 52 patients ( 27 males) underwent USAT for high Intermediate Risk PE. Patient characteristics are outlined in Table 2. 30 patients were unprovoked PE and 9 were provoked post surgeries and 11 patients had a diagnosis of cancer and 2 were on hormonal therapy. 11 had a history of prior VTE. The mean RV/ $\mathrm{LV}$ ratio on the CT was $1.52+0.36 .48$ patients had elevated cardiac biomarkers (Troponin I and/or B type Natiuretic Peptide). The 4 patients who did not were treated because of elevated PASP $>50$ and +DVT. 38 patients had initial RVSP $>50$ on initial echocardiogram. Lower extremity DVT was identified in 36 patients on the day of diagnosis by Ultrasound. Of these, 30 patients had a retrievable IVC filter placed. 30 patients met all 3 criteria listed in Table 1, and only 2 patients met 1 criteria (+troponin, criteria \#2).

Table 1: Clinical Criteria Characterizing High Intermediate Risk Pulmonary Embolism.

\begin{tabular}{|c|c|}
\hline Clinical Risk & Criteria \\
\hline \multirow{2}{*}{$\begin{array}{c}\text { Hemodynamic Deterioration } \\
\text { and/or Death }\end{array}$} & 1. RV $/ \mathrm{LV}>1.0$ on CT angiogram. PE in Main or Lobar arteries and lower extremity proximal DVT \\
\cline { 2 - 2 } CTEPH & 2. RV/LV>1.0 on CT angiogram. PE in Main or Lobar arteries and elevated cardiac biomarker \\
\hline
\end{tabular}

Note: Definition of abbreviations used: RV dilation = right ventricular/left ventricular $(\mathrm{RV} / \mathrm{LV})$ diameter ratio $>0.9$ on echocardiogram or CT scan; DVT = deep venous thrombosis. Cardiac biomarkers = troponin I and/or B-type natiuretic peptide; $\mathrm{RVSP}=$ right ventricular systolic pressure; $\mathrm{CTEPH}=$ chronic thromboembolic pulmonary hypertension.

Table 2: Study Population.

\begin{tabular}{|c|c|}
\hline Study Patient Characteristics & Study Patients $(n=52)$ \\
\hline Age, mean (SD), yr & $61(15)$ \\
\hline BMI, mean (SD) & $34(9)$ \\
\hline Male gender, $\mathrm{N}(\%)$ & $27(52)$ \\
\hline \multicolumn{2}{|c|}{ Medical comorbidities } \\
\hline Hypertension, n & 28 \\
\hline Recent Surgery, n & 9 \\
\hline History of cancer, n & 11 \\
\hline Prior VTE, $\mathrm{n}$ & 11 \\
\hline
\end{tabular}




\begin{tabular}{|c|c|}
\hline Asthma, $\mathrm{n}$ & 6 \\
\hline Hormonal therapy, $\mathrm{n}$ & 2 \\
\hline Cardiac disease, $\mathrm{n}$ & 3 \\
\hline Splenectomy, $\mathrm{n}$ & 1 \\
\hline \multicolumn{2}{|c|}{ Admission details } \\
\hline Troponin I peak (SD), ng/ml & $0.26(0.35)$ \\
\hline B-type natriuretic peptide (SD), pg/ml & $187(195)$ \\
\hline RV/LV ratio by CT (SD) & $1.52(0.36)$ \\
\hline Acute DVT, $\mathrm{n}$ & 36 \\
\hline IVC filter inserted, $\mathrm{n}$ & 30 \\
\hline Creatinine on day of USAT (SD), mg/dl & $1.05(0.8)$ \\
\hline Creatinine 48 hours post USAT (SD), $\mathrm{mg} / \mathrm{dl}$ & $0.97(0.7)$ \\
\hline
\end{tabular}

Note: Definition of abbreviations used: BMI = body mass index; VTE = venous thromboembolism; $\mathrm{RV} / \mathrm{LV}$ ratio by $\mathrm{CT}=$ right ventricle to left ventricle ratio by computerized tomography scan; DVT = deep venous thrombosis; IVC = inferior vena cava; USAT = catheter-directed ultrasound-accelerated thrombolysis.

\section{Procedural Details}

Bilateral catheters were utilized in 49 of 52 patients. Recombinant t-PA was infused at $0.5 \mathrm{mg} /$ hour/catheter in patients undergoing bilateral USAT (1.0mg/hour with unilateral USAT). The average r-tPA dose in our cohort was $22.1+4.9 \mathrm{mg}$. The average duration of USAT was $22.1+4.9$ hours. All patients received concurrent IV unfractionated heparin. The mean aPTT during thrombolytic infusion was $50.0+13.9$ seconds. Goal aPTT (4060 seconds) during thrombolytic infusion was achieved in $58 \%$ $(\mathrm{n}=30 / 52)$ patients.

\section{Change in Pulmonary Arterial Pressures}

Overall, there was a statistically significant reduction in the pulmonary artery systolic pressure and mean pulmonary artery pressure immediately following USAT. The pulmonary artery systolic pressure decreased $7.3 \%$ from $63.6+15.1 \mathrm{mmHg}$ to $59.0+$ $17.5 \mathrm{mmHg}$ ( $\mathrm{p}$ value $=0.0045)$. The mean pulmonary artery pressure decreased $5.4 \%$ from $37.5+8.0 \mathrm{mmHg}$ to $35.5+9.1 \mathrm{mmHg}$ (p value $=0.0097$ ). Thirty-eight patients had a PASP $>50 \mathrm{mmHg}$ at the time of initial catheterization. Within this subgroup, a $6.2 \%$ reduction in PASP was observed following USAT $(67.0+14.0 \mathrm{mmHg}$ to 62.9 $+16.6 \mathrm{mmHg}$; $\mathrm{p}$ value $=0.0023$ ). Only 7 patients had reductions in PASP < $50 \mathrm{mmHg}$ immediately following USAT despite a $53.1 \%$ reduction in Miller score $(20.0+3.9$ to $9.4+4.7$; p value $<0.0001)$.

\section{Change in Miller Score}

Miller scores were computed for 50 of 52 patients. One patient had a non-diagnostic pulmonary arteriography and another had imaging via carbon dioxide digital angiography, therefore she was excluded. There was a statistically significant decrease in the Miller score immediately following USAT. The Miller score decreased $55.0 \%$ (mean $19.7+3.8$ to $8.9+4.7 ;$ p value $<0.0001$ )

\section{Safety}

No procedural related complications occurred. There were no major or minor bleeding complications. No patient received blood transfusions.

\section{Mortality}

The in-hospital mortality rate for this cohort was $1.9 \%(n=1 / 52)$. The death was not procedural related. The patient underwent bilateral USAT. Despite a 52.9\% reduction in Miller score, pulmonary arterial pressures worsened (PASP $73 \mathrm{~mm} \mathrm{Hg}$ to $78 \mathrm{~mm} \mathrm{Hg}$; mean PAP $38 \mathrm{~mm} \mathrm{Hg}$ to $44 \mathrm{~mm} \mathrm{Hg}$ ). Three days after USAT, the patient suffered an acute neurological event. Emergent CT imaging revealed new pulmonary embolism with evidence of occlusive thrombus within the aortic arch extending into the proximal descending aorta, right and left common carotid, left middle cerebral, left subclavian, and left vertebral arteries. Lower extremity ultrasound from admission had revealed an acute popliteal DVT. IVC filter had not been placed. Transthoracic echocardiogram on the day of neurologic deterioration demonstrated a patent foramen ovale (PFO) and a non-occlusive thrombus within the IVC. Unfortunately, the patient progressed to brain death.

\section{Discussion}

Intermediate Risk PE accounts for over $30 \%$ of all hospitalized PE [26]. The in-hospital mortality rate in such patients is estimated at 2-3\% based on contemporary studies [5]. Despite the overall low mortality rate, there is substantial variability in clinical outcomes with some at risk for hemodynamic deterioration and some at risk for the development of CTEPH. To accept the bleeding risk of thrombolytic therapy, additional risk stratification is required. In this study, we defined high Intermediate Risk PE as the subset of patients that presented with acute symptoms and had CT angiographic 
evidence of clot within the central pulmonary circulation. These patients additionally demonstrated the combination of RV dilation (RV/LV>1.0 on CT scan) and elevated cardiac biomarkers (Table 1) with the additional findings of either proximal DVT or elevated RVSP estimated on cardiac echocardiogram. Systemic thrombolytic therapy, while beneficial in massive, High-Risk PE [15], has been associated with major bleeding complications in Intermediate Risk $\mathrm{PE}$, including intracranial hemorrhage [5].

USAT is an attractive treatment strategy for Intermediate Risk PE because it has the ability to rapidly reduce clot burden with a much greater safety profile than systemic thrombolysis [4,16-24]. Studies have consistently shown a reduction in $\mathrm{RV} / \mathrm{LV}$ ratio $[4,17,21-24]$ pulmonary arterial pressure $[4,16,18-24]$ and pulmonary arterial clot burden $[4,17,21-24]$ immediately following USAT. The recently published PERFECT registry [21] has both confirmed the safety of catheter directed therapies as well as demonstrating similar reductions in Pulmonary artery systolic pressure. Substantial variability exists in the published treatment protocols including those that use catheter directed therapy without ultrasound. Protocols have included upfront thrombolytic bolus as high as 8 $\mathrm{mg}$ of r-tPA, hourly catheter r-tPA doses from $0.5 \mathrm{mg}$ to $1 \mathrm{mg}$, with total $\mathrm{r}$-tPA doses ranging from $12 \mathrm{mg}$ to $35.1 \mathrm{mg}$ [4,16-24]. While all published reports are free of ICH, there continues to be major and minor bleeding complications [4,16-24]. In a recent review of all USAT studies, the rate of major bleeding complications was $3.6 \%$, with procedure related minor bleeding complications occurring in $10.7 \%$ [27].

In this report, a non-bolus, fixed, low dose, USAT protocol is utilized in the treatment of high Intermediate Risk PE. This protocol proved to be highly effective in reducing clot burden and acute pulmonary hypertension without bleeding related complications. We hypothesize that our excellent safety profile is related to the lack of upfront r-tPA bolus, the low dose hourly infusion rate of r-tPA, and the utilization of low dose IV unfractionated heparin for which an aPTT of 40-60 seconds is targeted during USAT. The inhospital mortality rate for this cohort was $1.9 \%(n=1 / 52)$. The one death was attributed to recurrent PE with paradoxical embolism through a documented Patent Foramen Ovale (PFO). The embolism unfortunately resulted in a catastrophic cerebral vascular accident. A PFO in PE is associated with an increased risk of complications [28]. During this period of study, we have incorporated several valuable components in our USAT protocol. First, all USAT candidates undergo transthoracic echocardiography with agitated saline to evaluate for clot within the right heart chambers and PFO. Secondly, all USAT candidates undergo bilateral lower extremity ultrasound for evaluation of proximal DVT.

Thirdly, at the time of catheter insertion, IVC venography is performed to evaluate for vena cava thrombus. Lastly, if large proximal lower extremity DVT is identified, retrievable IVC filter is considered. While much of the focus of USAT is rapid reversal of RV dysfunction, the long-term impact of USAT on RV function and development of a post PE syndrome or CTEPH is unknown. The development of CTEPH following an acute PE is believed to be uncommon, occurring at a rate of 0.5-4\% [29]. However, CTEPH may occur more frequently following a Intermediate Risk or High Risk PE [30]. Risk factors for CTEPH include large acute clot burden, systolic pulmonary artery pressure greater than $50 \mathrm{mmHg}$ at the time of diagnosis of acute embolism, and systolic pulmonary artery pressure greater than $50 \mathrm{~mm} \mathrm{Hg}$ at the time of hospital discharge [8-14,30,31]. Recent studies have suggested that USAT in Intermediate Risk PE may reduce persistent RVD and pulmonary hypertension when compared to standard anticoagulation therapy alone $[9,18]$. Thirty-eight in this cohort had PASP > $50 \mathrm{mmHg}$ by initial catheterization, 31 of which had persistent elevation in PASP $>50 \mathrm{mmHg}$ at the conclusion of USAT.

Persistent pulmonary hypertension following USAT may be a marker of pre-existing pulmonary hypertension or a risk factor for the development of CTEPH. One advantage of our protocol is that we were able to directly assess clot burden reduction, but this did not correlate with pulmonary pressure reductions. The $55 \%$ reduction in Miller Index is similar to reported reductions from systemic thrombolysis [25]. The lack of correlation between clot reduction and Pulmonary pressures may have occurred because we did not account for cardiac output improvement or calculate PVR. Other possibilities to explain this discrepancy includes an element of acute pulmonary vasoconstriction, already the establishment of CTEPH or other causes of pre-existing pulmonary hypertension. The main limitations of this study include the retrospective single arm analysis with small sample size. Although this standardized protocol demonstrated safety and efficacy, the optimal infusion rate, total dose of thrombolytic and duration of USAT remains unanswered.

A randomized trial designed to determine the optimal dose of thrombolytic and duration of the ultrasound procedure as a treatment for Intermediate Risk PE (OPTALSE PE) has been published [32,33]. Our experience with a lower infusion dose of TPA gives further support to the safety of the procedure. Lastly, this study lacks long-term follow up, and therefore, conclusions on the impact of USAT on the development or prevention of the post PE syndrome or CTEPH cannot be drawn. Our selection criteria of utilizing USAT on cases that presented with elevated RVSP may have biased our results with a group of patients that already have pulmonary hypertension explaining the limited reduction in PAP at 24 hrs compared to prior trials [21,23]. In conclusion, this study adds to the growing body of evidence that USAT with concurrent low dose heparin is safe and effective in reducing acute 
pulmonary hypertension and clot burden in high Intermediate Risk PE. This protocol incorporates additional considerations for the use of USAT in Intermediate Risk PE; specifically, lower extremity ultrasonography, initial RVSP $>50 \mathrm{~mm} \mathrm{Hg}$, IVC venography, and contrast echocardiography. These concepts should be considered for future study design protocols. Further studies are required to define the role of USAT in the treatment of High Intermediate Risk $\mathrm{PE}$ and the impact on the post PE syndrome and CTEPH.

\section{Acknowledgment}

The authors would like to thank Doctor Daniel J Sheibley and Rebecca Kwait for their invaluable support. Drs. Greenspon,Reber,Villas and Bonn are responsible for the design , data collection, and implementation of this study. Drs. Desai, Plush, and Hegewald were responsible for Data collection. Dr. Rochani was responsible for the statistical analysis. Drs. Greenspon and Reber are responsible for the manuscript preparation.

\section{Funding}

No source of funding.

\section{Conflicts of Interest}

Lee W Greenspon and Joseph Bonn were co-investigators for the OPTALYSE (NCT02396758) Investigational Research Study supported by the BTG Corporation.

\section{Summary Statement}

A low dose TPA of $0.5 \mathrm{mg} /$ catheter/hr during catheter directed Ultrasound accelerated thrombolysis is safe and effective in High Intermediate Risk Pulmonary Embolism.

\section{References}

1. Wood KE (2002) Major pulmonary embolism: review of a pathophysiologic approach to the golden hour of hemodynamically significant pulmonary embolism. CHEST Journal 121(3): 877-905.

2. Jaff MR, McMurtry MS, Archer SL, R James White, Brenda K Zierler, et al. (2011) Management of massive and submassive pulmonary embolism, iliofemoral deep vein thrombosis, and chronic thromboembolic pulmonary hypertension a scientific statement from the American Heart Association. Circulation 123(16): 1788-1830.

3. Kasper W, Konstantinides S, Geibel A, M Redecker, J Kienast, et al. (1997) Management strategies and determinants of outcome in acute major pulmonary embolism: results of a multicenter registry. Journal of the American College of Cardiology 30(5): 1165-1171.

4. Kennedy RJ, Kenney HH, Dunfee BL (2013) Thrombus resolution and hemodynamic recovery using ultrasound-accelerated thrombolysis in acute pulmonary embolism. Journal of Vascular and Interventional Radiology 24(6): 841-848.

5. Meyer G, Vicaut E, Danays T, Cecilia Becattini, Erich Bluhmki, et al. (2014) Fibrinolysis for patients with intermediate-risk pulmonary embolism. New England Journal of Medicine 370(15): 1402-1411.

6. Konstantinides S, Geibel A, Heusel G, Fritz Heinrich, Wolfgang Kasper, et al. (2002) Heparin plus alteplase compared with heparin alone in patients with submassive pulmonary embolism. New England Journal of Medicine 347(15): 1143-1150.
7. Jiménez D, Aujesky D, Moores L, Wolfgang Kasper (2011) Combinations of prognostic tools for identification of high-risk normotensive patients with acute symptomatic pulmonary embolism. Thorax 66(1): 75-81.

8. Stein PD, Matta F, Janjua M, Fadel Jaweesh, Ahmed Alrifai, et al. (2010) Outcome in stable patients with acute pulmonary embolism who had right ventricular enlargement and/or elevated levels of troponin I. The American journal of cardiology 106(4): 558-563.

9. Fasullo S, Scalzo S, Maringhini G, Salvatore Paterna, Pietro Di Pasquale, et al. (2011) Six-month echocardiographic study in patients with submassive pulmonary embolism and right ventricle dysfunction: comparison of thrombolysis with heparin. The American journal of the medical sciences 341(1): 33-39.

10. Pepke Zaba J, Delcroix M, Lang I, Paul Bresser, Adam Torbicki, et al. (2011) Chronic thromboembolic pulmonary hypertension (CTEPH) results from an international perspective registry. Circulation 124(18): 1973-1981.

11. Sharifi M, Bay C, Skrocki L (2013) Moderate pulmonary embolism treated with thrombolysis (from the "MOPETT" Trial). The American journal of cardiology 111(2): 273-277.

12. Stevinson BG, Hernandez-Nino J, Rose G (2007) Echocardiographic and functional cardiopulmonary problems 6 months after first-time pulmonary embolism in previously healthy patients. European heart journal 28(20): 2517-2524.

13. Hoeper MM, Mayer E, Simonneau G (2006) Chronic thromboembolic pulmonary hypertension. Circulation 113(16): 2011-2020.

14. Ribeiro A, Lindmarker P, Johnsson H (1999) Pulmonary embolism oneyear follow-up with echocardiography Doppler and five-year survival analysis. Circulation 99(10): 1325-1330.

15. Stein PD, Matta F (2012) Thrombolytic therapy in unstable patients with acute pulmonary embolism: saves lives but underused. The American journal of medicine 125(5): 465-470.

16. Bagla S, Smirniotopoulos JB, Van Breda A, Sheridan MJ, Sterling KM (2015) Ultrasound-Accelerated Catheter-Directed Thrombolysis for Acute Submassive Pulmonary Embolism. Journal of Vascular and Interventional Radiology 26(7): 1001-1006.

17. Dumantepe M, Uyar I, Teymen B, Ugur O, Enc Y (2014) Improvements in Pulmonary Artery Pressure and Right Ventricular Function After Ultrasound-Accelerated Catheter-Directed Thrombolysis for the Treatment of Pulmonary Embolism. Journal of cardiac surgery 29(4): 455-463.

18. Engelberger RP, Moschovitis A, Fahrni J, Iris Baumgartner, Nils Kucher, et al. (2013) Fixed low-dose ultrasound-assisted catheter-directed thrombolysis for intermediate-and high-risk pulmonary embolism. European heart journal 36(10): 597-604.

19. Engelhardt TC, Taylor AJ, Simprini LA, Kucher N (2011) Catheterdirected ultrasound-accelerated thrombolysis for the treatment of acute pulmonary embolism. Thrombosis research 128(2): 149-154.

20. Kucher N, Boekstegers P, Muller O, Klaus Empen, Iris Baumgartner, et al. (2014) Randomized, Controlled Trial of Ultrasound-Assisted CatheterDirected Thrombolysis for Acute Intermediate-Risk Pulmonary Embolism. Circulation 129(4): 479-486.

21. Kuo WT, Banerjee A, Kim PS, Miguel A De Gregorio, Michael J Hall, et al. (2015) Pulmonary Embolism Response to Fragmentation, Embolectomy, and Catheter Thrombolysis (PERFECT): Initial Results from a Prospective Multicenter Registry. CHEST Journal 148(3): 667-673.

22. McCabe JM, Huang PH, Riedl L, Eisenhauer AC, Sobieszczyk P (2015) Usefulness and Safety of Ultrasound-Assisted Catheter-Directed Thrombolysis for Submassive Pulmonary Emboli. The American journal of cardiology 115(6): 821-824. 
23. Piazza G, Hohlfelder B, Jaff MR, Samuel Z, Goldhaber et al. (2015) A prospective, single-arm, multicenter trial of ultrasound-facilitated, catheter-directed, low-dose fibrinolysis for acute massive and submassive pulmonary embolism: The SEATTLE II study. JACC: Cardiovascular Interventions 8(10): 1382-1392.

24. Quintana D, Salsamendi J, Fourzali R, Narayanan G (2014) Ultrasoundassisted thrombolysis in submassive and massive pulmonary embolism: assessment of lung obstruction before and after catheter-directed therapy. Cardiovascular and interventional radiology 37(2): 420-426.

25. Miller G, Sutton G, Kerr I, Gibson R, Honey M (1971) Comparison of streptokinase and heparin in treatment of isolated acute massive pulmonary embolism. BMJ 2(5763): 681-684.

26. Grifoni S, Olivotto I, Cecchini P, G Agnelli, G Berni, et al. (2000) Short-term clinical outcome of patients with acute pulmonary embolism, normal blood pressure, and echocardiographic right ventricular dysfunction. Circulation 101(24): 2817-2822.

27. Engelberger RP, Kucher N (2014) Ultrasound-assisted thrombolysis for acute pulmonary embolism: a systematic review. European heart journal 35(12): 758-764.

28. Doyen D, Castellani M, Moceri P, Claire Chaussade, Emile Ferrari, et al. (2014) Patent foramen ovale and stroke in intermediate-risk pulmonary embolism. CHEST Journal 146(4): 967-973.

\section{ISSN: 2574-1241}

DOI: 10.26717/BJSTR.2021.38.006094

Lee W Greenspon. Biomed J Sci \& Tech Res

(c) (P) This work is licensed under Creative BY Commons Attribution 4.0 License

Submission Link: https://biomedres.us/submit-manuscript.php
29. Pengo V, Lensing AW, Prins MH, Antonio Marchiori, Bruce L Davidson, et al. (2004) Incidence of chronic thromboembolic pulmonary hypertension after pulmonary embolism. New England Journal of Medicine 350(22): 2257-2264

30. Kline JA, Steuerwald MT, Marchick MR, Geoffrey A Rose (2009) Prospective evaluation of right ventricular function and functional status 6 months after acute submassive pulmonary embolism: frequency of persistent or subsequent elevation in estimated pulmonary artery pressure. CHEST Journal 136(5): 1202-1210.

31. Guerin L, Couturaud F, Parent F (2014) Prevalence of chronic thromboembolic pulmonary hypertension after acute pulmonary embolism. Thromb Haemost 112(3): 598-605.

32. Tapson VF, Sterling k, Jones N, Elder M, Tripathy U, et al. (2018) A randomized Trial of the Optimum Duration of Acoustic Pulse Thrombolysis Procedure in Acute Intermediate Risk Pulmonary Embolism (The OPTALYSE PE Trial). JACC: Cardiovascular Interventions 11: $1401-1410$

33. Fedullo P, Kerr KM, Kim NH, William R, Auger (2011) Chronic thromboembolic pulmonary hypertension. American journal of respiratory and critical care medicine 183(12): 1605-1613.

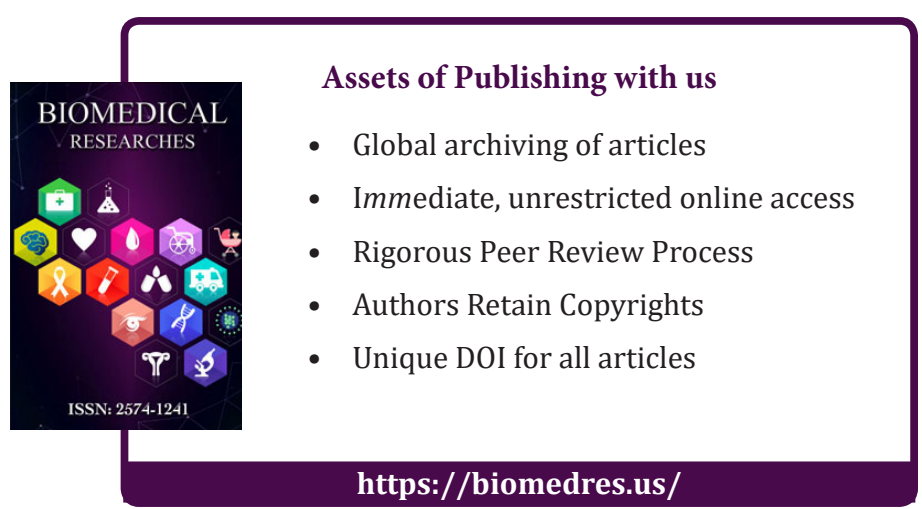

